\title{
Do guns kill people or...? The mesh dilemma
}

\author{
Charles W. Butrick
}

Received: 23 November 2009 / Accepted: 9 December 2009 / Published online: 9 January 2010

(C) The International Urogynecological Association 2010

Many of the articles in this journal and others demonstrate the mixed messages of mesh augmented repairs for pelvic organ prolapse. At this point, short-term data (2-year follow-up or less) suggest that its use for anterior compartment defects may provide an advantage over nonmesh augmented repairs [1, 2], but at what cost [3, 4]?

Complications are certainly possible with any surgical procedure; but one of the most concerning complications that can occur is worsening or de novo postoperative pain disorders. But are the complications of postoperative pain caused by the mesh kits or are they caused by the physicians who are using them?

I see many patients in my office sent from various physicians located throughout the Midwest with problems of postoperative pain after a mesh-augmented transvaginal repair for prolapse. Almost universally, these patients had a pelvic pain disorder that was either overt (i.e., patients who had prolapse surgery done because of the chief complaint of pelvic pain described as pressure in the absence of prolapse being present at the introitus or beyond) or had a more subtle pelvic pain disorder. These subtle pelvic pain disorders are often compensated for or tolerated by the patient for many years. These patients often have a history that is compatible with primary dyspareunia or dysmenorrhea, vulvodynia, chronic constipation, painful bladder syndrome, or fibromyalgia prior to coming to these physicians asking for surgery to be done for prolapse. Clinicians must realize that $50-80 \%$ of patients with these pelvic pain disorders have hypertonic pelvic floor dysfunction and/or myofascial pain [5]. Attaching a piece of

\section{W. Butrick $(\bowtie)$}

The Urogynecology Center,

12200 W 106th, Suite 130,

Overland Park, KS 66215, USA

e-mail: cwbutrick@gmail.com mesh that then supports the prolapsed viscera at a single or possibly four sites of attachments to these muscles that are already spastic, hypertonic, and demonstrating dysfunction and allodynia simply exacerbates and perpetuates their chronic pain disorders.

We must remember that prolapse does not cause pelvic pain, especially when symptoms seem greater than the degree of prolapse seen. If a patient has a history or findings compatible with pelvic floor tension myalgia and/or hypertonic dysfunction we must avoid doing a surgery that involves attaching or anchoring the support of the prolapsed organs to these muscles. This statement should not be surprising to any clinician involved in the repair of pelvic organ prolapse because we all know that procedures like sacro-spinous vaginal vault suspensions and posterior repairs done by techniques that involve levatorplasty are often associated with both short-term and long-term pain and often times dyspareunia for many years postoperatively.

While these mesh kit procedures all involve attachment to the pelvic floor muscles to anchor the entire repair, not all patients who undergo this procedure develop a significant postoperative pelvic pain disorder. The use of muscle sites seems to be intuitively a bad idea, but many of our patients tolerate this non-physiologic but anatomically correct repair without problems. I contend that it is not the mesh kit but the patient selection that is the key to preventing the induction of chronic myofascial pain and at times causing these patients to become a pelvic cripple; unable to stand, sit, or have intercourse.

Therefore, do guns kill people? No, people kill people. So we must avoid anchoring prolapsed organs to a few muscle sites in patients with pre-existing myofascial dysfunction and/or pelvic pain. These patients will be better served supporting their prolapse to the uterosacral ligaments or the longitudinal ligament overlying the sacrum 
(i.e., sacrocolpopexy). This editorial does not address the other issues of importance in helping our patients achieve a good outcome. These other issues include physician experience, training, and the ability for a physician to make appropriate decisions and be able to bring to the operating room the surgical abilities to repair prolapse with a multitude of surgical options that he/she is comfortable with (not just a "one size fits all" approach). We must therefore tailor an approach with the patient's needs, fears, and history all being considered. In select patients, transvaginal mesh-augmented repairs are truly a real advance in the management of our patients at high risk for failure, but should not be used in all patients with prolapse. The consistent use of regional anesthesia both intraoperatively and postoperatively as well as aggressive treatment of myofascial pain, if it should occur postoperatively, will benefit your patients and your own peace of mind.

\section{References}

1. Carey M, Higgs P, Goh J, Lim J, Leong A, Krause H, Cornish A (2009) Vaginal repair with mesh versus colporrhaphy for prolapse: a randomised controlled trial. BJOG 116:1380-1386

2. Hiltunen R, Nieminen K, Takala T, Heiskanen E, Merikari M, Niemi K, Heinonen PK (2007) Low-weight polypropylene mesh for anterior vaginal wall prolapse: a randomized controlled trial. Obstet Gynecol 110:455-462

3. Lewicky-Gaupp C, McGuire EJ, Fenner DE (2009) Multiple perineal abscesses and sinus tracts as a complication of vaginal mesh. Int Urogynecol J Pelvic Floor Dysfunct 20:1137-1139

4. Altman D, Elmer C, Kiilholma P, Kinne I, Tegerstedt G, Falconer C (2009) Sexual dysfunction after trocar-guided transvaginal mesh repair of pelvic organ prolapse. Obstet Gynecol 113:127133

5. Butrick CW, Sanford D, Hou Q, Mahnken JD (2009) Chronic pelvic pain syndromes: clinical, urodynamic, and urothelial observations. Int Urogynecol J Pelvic Floor Dysfunct 20:10471053 